\title{
Degradation Studies of InAs / GaAs QD Lasers grown on Si
}

\author{
Samuel Shutts ${ }^{1}$, C.P. Allford ${ }^{1}$, C.Spinnler ${ }^{2}$, Z. Li ${ }^{1}$, M. Tang ${ }^{3}$ and H Liu ${ }^{3}$, P. M. Smowton ${ }^{1}$ \\ ${ }^{I}$ School of Physics and Astronomy, Cardiff University, Cardiff, CF24 3AA, U.K. \\ ${ }^{2}$ University of Basel,Basel, Suisse \\ ${ }^{3}$ Department of Electronic and Electrical Engineering, University College London, London WC1E 7JE, UK \\ ShuttsS@cf.ac.uk,SmowtonPM@cf.ac.uk
}

Abstract: Lowering the threshold gain of InAs quantum dot lasers grown on Silicon, significantly extends device lifetime. Measurements on degraded devices show increased optical mode loss is responsible for degradation and a consequent shortening of lasing wavelength.

Reliable and efficient electrically-pumped silicon-based lasers are currently required as sources in silicon photonic integrated circuits and ultimately to enable full integration of photonics and electronics. While wafer bonding of Compound Semiconductors (CS) and Silicon is the favoured immediate solution, in the longer term epitaxial growth of CS on silicon is seen as the ideal for large scale manufacturing. Recent results using Quantum Dots (QDs) show promising lifetimes [e.g. [1] [2]] even though epitaxially grown CS Quantum well $(\mathrm{QW})$ laser on Si structures degrade within seconds. It is understood that both the number and size of defects present in a laser structure can increase during operation and this in-turn degrades performance and ultimately shortens the device lifetime. In QW lasers it has been shown that the primary mechanism for degradation is the increased non-radiative recombination rate that accompanies the defects, although an increased internal optical mode loss can also contribute. QDs bring two major advantages, firstly, they can effectively deflect defects or pin them preventing loop formation, and secondly, they localise charge carriers preventing them from diffusing laterally and recombining non-radiatively at a defect site. The latter, reduces the rate of degradation because it suppresses recombination enhanced defect reactions (REDR), a process which can cause dislocation climb, further reducing device performance. QDs are effective at isolating carriers and limiting the recombination at defect sites, however, the optical mode propagates along the entire length of the laser cavity and therefore will always suffer from the increase in dislocations via optical scattering, characterised by the internal optical mode loss. In this study, we test the reliability of deep-etched ridgewaveguide QD lasers grown directly on Si substrates and investigate how factors such as cavity length, and hence carrier density, affect degradation rate whilst running in continuous wave $(\mathrm{CW})$ operation. Using a segmented contact structure, that can be run as either a laser or a single-pass gain structure, we show that measured optical mode loss increases for devices on life test and is a major contributing factor on the eventual degradation of performance in these QD-on-Si lasers.

The samples examined here are similar designs to those used in reference [1], but with a larger dot size distribution and consequently somewhat higher threshold current density for similar cavity lengths. However, most importantly the approaches to minimise defects propagating towards the active region, comprising 5 layers of InAs dots each grown in a dot-in-a-well (DWELL) on $\operatorname{In}_{0.15} \mathrm{Ga}_{0.85} \mathrm{As}$ surrounded by a GaAs core, are similar. These include $\mathrm{Si}(100)$ wafers with $4^{\circ}$ offcut to the [011] plane, on which a thin nucleation layer made of AlAs was deposited by migration enhanced epitaxy using alternating $\mathrm{Al}$ and $\mathrm{As}_{4}$ flux at a low growth temperature of $350{ }^{\circ} \mathrm{C}$, then three layers of GaAs grown at $350{ }^{\circ} \mathrm{C}, 450{ }^{\circ} \mathrm{C}$, and $590^{\circ} \mathrm{C}$ for $30 \mathrm{~nm}, 170 \mathrm{~nm}$, and $800 \mathrm{~nm}$, respectively and strained layer superlattices (SLSs) grown as dislocation filter layers (DFLs). The structures are represented in Figure 1a. We expect these samples to have a low density of threading dislocations at the active region of on the order of $10^{5} \mathrm{~cm}^{-2}$ as has been observed using TEM in samples incorporating a similar layer structure. Ridge waveguides, shown schematically in Fig. 1b were dry-etched to a depth of approximately 3.2 microns using an Oxford Instruments Plasmalab 100. The epi-side n-contact ( $\mathrm{AuGe} / \mathrm{Ni} / \mathrm{Au}$ ) was deposited and annealed prior to planarising with Benzocyclobutene (BCB) and later reexposed by back-etching the $\mathrm{BCB}$ using reactive ion etching (RIE) with $\mathrm{C}_{4} \mathrm{~F}_{8} / \mathrm{O} 2$ plasma before final curing at $300^{\circ} \mathrm{C}$. Cr/Au was deposited for the p-contact to a thickness of 10 and $400 \mathrm{~nm}$ respectively. Cleaved-facet devices are produced by first thinning the Si substrate by lapping. Devices are mounted on T08 transistor headers epi-side up using a silver-loaded epoxy resin and each laser wire bonded using $30 \mu \mathrm{m}$ wires. It is appreciated that this method of mounting is not optimized for heat extraction and therefore limits the performance of the devices when operating $\mathrm{CW}$. The power-current (P-I) characteristics for a typical $10 \mu \mathrm{m} \times 3000 \mu \mathrm{m} \mathrm{CW}$ operated laser are shown in Fig. 2a as a function of temperature. The inset shows the lasing wavelength measured $10 \%$ above threshold at $21^{\circ} \mathrm{C}$ and has a peak value of $1297 \mathrm{~nm}$. There is a characteristic thermal roll-over in the power level as the current is increased, which is evident when we compared to pulsed operation at the same heat-sink temperature, as illustrated in Fig. 2b. As expected, there is also an increase in threshold current in CW operation due to the effects of self-heating. When performing $\mathrm{CW}$ measurements, there is a finite amount of time for self-heating to take full effect as the current increases and therefore the P-I data was taken in a quasi-steady state with a step rate of $\sim 1.4 \mathrm{mAs}^{-1}$.

Lasers with different cavity lengths have different threshold gain and different threshold carrier density and so we tested lasers with cavity lengths of 2000, 3000 and $4000 \mu \mathrm{m}$. Each device was operated CW at a constant current (1.3 times threshold) with the light output, voltage and temperature monitored at 5 minute intervals. Testing was only interrupted to perform light-current-voltage (L-I-V) and wavelength measurements. The 
experiment revealed a reduction in the light output for lasers of all cavity lengths, with degradation occurring more rapidly for lasers with shorter cavity lengths (a greater fractional loss in light output with time). Threshold current values (Jth) extracted from interval L-I measurements, plotted in figure 3, show an increase in threshold current with aging, which is significant for the shortest $(2000 \mu \mathrm{m})$ laser which failed to operate $\mathrm{CW}$ beyond 1000 hours. As aging proceeds, the external differential efficiency of the lasers also reduced, with the most significant fall occurring within the first few hundred hours of operation as shown in the insert of figure 3 for a $4000 \mu \mathrm{m}$ long laser. Another $4000 \mu \mathrm{m}$ laser was operated at 3.0 times threshold to observe how the increase in current density, for a given cavity length, would affect degradation rate. Despite the higher current density, which was comparable to the operating current density of the $2000 \mu \mathrm{m}$ laser, the degradation rate did not increase significantly. The reason for this, is that above threshold the stimulated emission process causes rapid recombination of carriers in the active region of the laser and therefore, does not significantly increase the available carriers which can recombine by non-radiate processes, via a defect site.. The consequence of this, is that degradation is not so affected by operating current density, but rather the current density required to reach threshold. The experimental optical loss data has been obtained by the electrically pumped segmented contact method [3]. The early on-set of increased modal-loss is illustrated in figure 4, where the modal absorption is plotted as a function of wavelength before and after CW operation of the device. The modal-loss includes inter-band absorption of the inhomogeneously broadened dot states and the internal optical mode loss $\left(\alpha_{\mathrm{i}}\right)$ (identified by the dashed lines), observable below the absorption wavelengths of the dots. The internal optical loss increased from $2.77+/-0.09 \mathrm{~cm}^{-1}$ to $3.15+/-0.08 \mathrm{~cm}^{-1}$. This is consistent with the blue-shift we observe for laser wavelength following life-tests.

In summary InAs QD ridge-waveguide lasers of different length grown directly on Si substrates have been operated at constant current and interval measurements of L-I-V characteristics used to determine changes in threshold current and slope efficiency. The rate of degradation depended on the cavity length of the laser being tested, revealing that the lower the operating carrier density, the lower the rate of degradation. Measurements also reveal that optical mode loss increases with time on test.

This research is based on work supported by the UK Engineering Physical Sciences Research Council (EPSRC) under grant number EP/P006973/1.

a
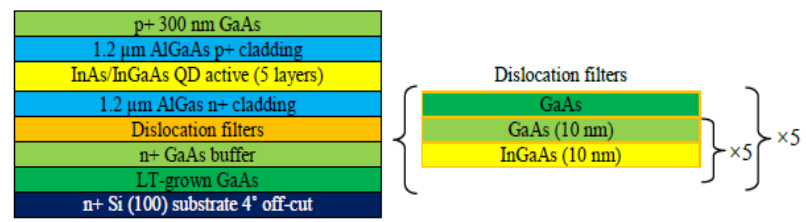

b

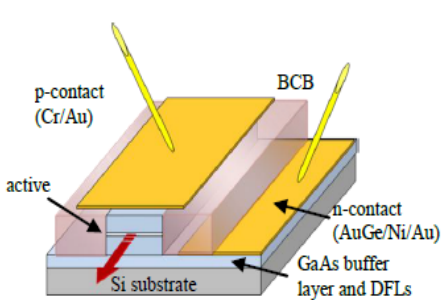

Fig. 1: (a) Schema of epitaxial growth structure including details of defect filters and (b) Ridge-waveguide on Si substrate.
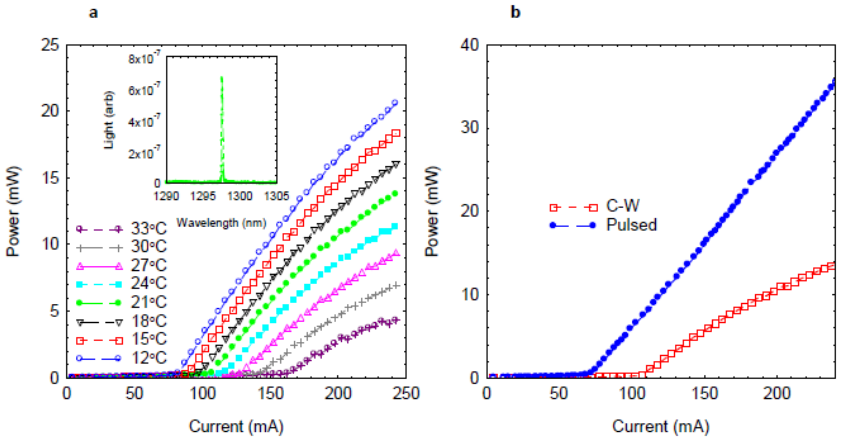

Fig. 2 (a) Power-current characteristics vs. temperature for a $10 \mu \mathrm{m} \times 3000$ $\mu \mathrm{m}$ laser operating CW. Inset: shows lasing wavelength at $21^{\circ} \mathrm{C}$. (b) Power-current curves for $10 \mu \mathrm{m} \times 3000 \mu \mathrm{m}$ laser operated pulsed and $\mathrm{CW}$.

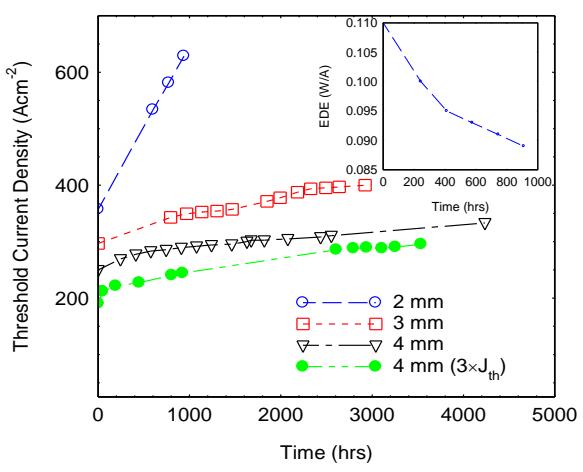

Fig. 3: Threshold current density versus $\mathrm{CW}$ operating time for lasers of different cavity length. Inset showing external differential efficiency (EDE) vs. time for a $4 \mathrm{~mm}$ laser aged at 1.3 times threshold.

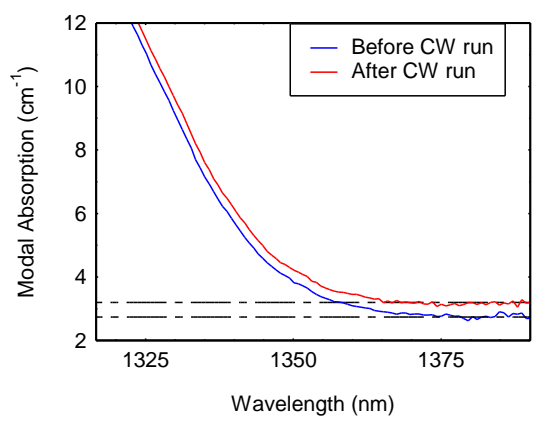

Fig. 4: Modal loss vs. wavelength showing absorption due to the dot states and the observable region of the internal optical mode loss $\left(\alpha_{\mathrm{i}}\right)$ The loss has shifted from $2.77+/-0.09 \mathrm{~cm}^{-1}$ to $3.15+/-0.08 \mathrm{~cm}^{-1}$.

[1] S. Chen et al., "Electrically pumped continuous-wave III-V quantum dot lasers on silicon," Nat. Photonics, 10, 5, 307-311, 2016.

[2] D. Jung et al., "Impact of threading dislocation density on the lifetime of InAs quantum dot lasers on Si," Appl. Phys. Lett., 112, 15, $153507,2018$.

[3] P. Blood et al "Characterization of Semiconductor Laser Gain Media by the Segmented Contact Method," 9, 5, 1275-1282, 2003. 